\section{BRAZIULIAN JOURNAL}

OF MEDICAL AND BIOLOGICAL RESHARCH

www.bjournal.com.br
ISSN 0100-879X

Volume 43 (01) 1-123 January 2010

BIOMEDICAL SCIENCES

AND

CLINICAL INVESTIGATION

Braz J Med Biol Res, J anuary 2010, Volume 43(1) 96-99

\title{
Do Caucasian and Asian clocks tick differently?
}

A.A. Barbosa, M. Pedrazzoli, B.D.V. Koike and S. Tufik

The Brazilian Journal of Medical and Biological Research is partially financed by
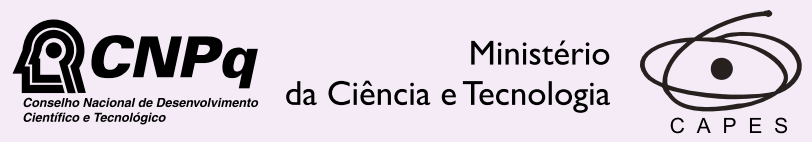

Ministério da Educação

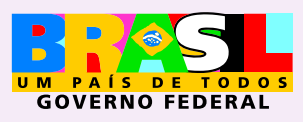

Institutional Sponsors 


\title{
Do Caucasian and Asian clocks tick differently?
}

\author{
A.A. Barbosa1 ${ }^{1}$, M. Pedrazzoli ${ }^{1,2}$, B.D.V. Koike ${ }^{1}$ and S. Tufik ${ }^{1}$ \\ 1Departamento de Psicobiologia, Instituto do Sono, \\ Universidade Federal de São Paulo, São Paulo, SP, Brasil \\ ${ }^{2}$ Curso de Gerontologia, Escola de Artes, Ciências e Humanidades, \\ Universidade de São Paulo, São Paulo, SP, Brasil
}

\begin{abstract}
The Period 3 and Clock genes are important components of the mammalian molecular circadian system. Studies have shown association between polymorphisms in these clock genes and circadian phenotypes in different populations. Nevertheless, differences in the pattern of allele frequency and genotyping distribution are systematically observed in studies with different ethnic groups. To investigate and compare the pattern of distribution in a sample of Asian and Caucasian populations living in Brazil, we evaluated two well-studied polymorphisms in the clock genes: a variable number of tandem repeats (VNTR) in PER3 and a single nucleotide polymorphism (SNP) in CLOCK. The aim of this investigation was to search for clues about human evolutionary processes related to circadian rhythms. We selected 109 Asian and 135 Caucasian descendants. The frequencies of the shorter allele (4 repeats) in the PER3 gene and the T allele in the CLOCK gene among Asians (0.86 and 0.84 , respectively) were significantly higher than among Caucasians ( 0.69 and 0.71 , respectively). Our results directly confirmed the different distribution of these polymorphisms between the Asian and Caucasian ethnic groups. Given the genetic differences found between groups, two points became evident: first, ethnic variations may have implications for the interpretation of results in circadian rhythm association studies, and second, the question may be raised about which evolutionary conditions shaped these genetic clock variations.
\end{abstract}

Key words: PER3 gene; CLOCK gene; Circadian rhythms; Asian; Caucasian; Ethnic

\section{Introduction}

Clock genes are a group of genes involved in the regulation of circadian rhythms such as the sleep/wake cycle, cortisol hormone secretion and core body temperature. These rhythms play important roles as physiological processes that determine adaptation to the environmental light/dark cycle. The entrainment by light in mammals is mainly orchestrated by the master circadian pacemaker located in the suprachiasmatic nuclei of the hypothalamus $(1,2)$.

In the suprachiasmatic nuclei, the molecular clock machinery works through a core feedback loop of the transcriptiontranslation of the PER1, PER2, PER3, CRY1, CRY2, CLOCK, $T I M$, and BMAL1 genes. These clock genes are expressed robustly in the $\mathrm{SNC}$ region, but they are also widely expressed in different tissues of the human body as tissue-specific cellular clocks $(1,2)$.

Among these clock genes, PER3 is of special interest since it contains a rare polymorphism in the coding region of the human genome, a variable number of tandem repeats
(VNTR) with four or five copies of a 54-bp sequence at exon 18 , which has recently been reported to be associated with delayed sleep phase syndrome $(1,2)$

In man and other animals, mutations and polymorphisms in a number of these core clock genes have been shown to alter circadian parameters. These variations are considered to be very promising genetic markers for understanding the role of clock genes in behavior and health. Several studies have shown that functional variations in clock genes are associated with diurnal preference ("morningness" and "eveningness") and sleep-phase syndromes in man (3-6).

In spite of numerous reports of association between circadian phenotypes and clock gene polymorphisms in Asian and Caucasian populations, little attention has been given to the fact that significant ethnic differences in the distribution of these genetic variations do exist. For example, polymorphic loci in one ethnic group have been described as non-polymorphic or with very different allelic frequencies in another $(5,7)$.

Correspondence: M. Pedrazzoli, Departamento de Psicobiologia, UNIFESP, Rua Napoleão de Barros, $925,3^{\circ}$ andar, 04024-002 São Paulo, SP, Brasil. Fax: +55-11-5572-5092. E-mail: pedrazzo@usp.br

Received May 19, 2009. Accepted November 16, 2009. Available online December 7, 2009. Published January 11, 2010. 
Another typical example of differences between Caucasians and Asians is a polymorphism characterized by the change of a nucleotide $\mathrm{T}$ to $\mathrm{C}$ at position 3111 (T3111C) of the circadian gene CLOCK. Several studies have shown associations or not with circadian phenotypes, but in spite of the controversy about the effect of this polymorphism in circadian regulation, when one analyzes the allelic frequencies in these five different studies $(4,6,8-10)$ conducted in different laboratories, it is possible to observe that the intragroup allelic frequencies are similar in Caucasian or Asian studies, but different between the ethnic groups studied.

Other studies with clock-related genes have also shown differences between ethnic groups. The G619A polymorphism in the AANAT gene related to melatonin synthesis has been reported to be associated with delayed sleep phase syndrome in an Asian population (11), but it was almost absent in Caucasians (12). The S408N polymorphism in the casein kinase $\varepsilon$ protein has been reported to be associated with delayed sleep phase syndrome in a Japanese population by Takano et al. (5), but was practically not found in a predominantly Caucasian sample (7); more specifically, while Takano et al. (5) found an allelic frequency of 0.12 for the N408 polymorphism in a Japanese population, Castro et al. (7) found a frequency of 0.007 in a Brazilian sample, showing that this polymorphism is very rare in the Brazilian population.

As clock gene function is an important part of the entrainment process to environmental light/dark conditions, which by itself varies greatly during the year in a latitude-dependent manner, the genetic variation present in different populations could reflect selection forces acting in populations living under different conditions of natural lighting.

The Brazilian population consists mainly of European (Portuguese) and native Brazilian backgrounds, later mixed with a variety of African groups and, at the beginning of the 20th century, with a variety of European ethnic groups (mainly Italian and Spanish) and Asian ethnic groups (mainly Japanese) living in tropical areas with particular light conditions (13). However, despite this miscegenation, since the last migration flow was very recent, it is still possible to select unmixed Asian and Caucasian individuals. This diversity makes this population highly suitable for ethnic genetic studies.

Thus, our objective was to compare the frequency of the VNTR polymorphism of PER3 and T3111C of the CLOCK genes in two population samples of Asian and Caucasian ethnic backgrounds living in Brazil with the intention to find clues that could contribute to constructing a new view about the human evolutionary process related to circadian rhythms.

\section{Material and Methods}

\section{Subjects and ethnic classification \\ We selected Asian and Caucasian volunteers among}

university students living in São Paulo, Brazil. The appearance of the subjects was observed visually, and a questionnaire that determines ancestry was administered. The subjects were asked about the two preceding generations in their families and reported the countries of origin and ethnicity of their parents and grandparents (online material) and themselves. We selected only those people of full Asian or Caucasian ancestry. The study was approved by the Ethics Committee of Universidade Federal de São Paulo and an informed consent was obtained from the participants. A total of 109 Asian (76 females and 33 males) and 135 Caucasian (65 females and 70 males) volunteers, mean age $28.5 \pm 14.7$ years, participated in the study.

\section{DNA collection}

DNA samples were collected from the buccal cells using a cytobrush. The samples were collected from all participants in the study, and DNA was extracted using the commercial Illustra blood genomic Prep Mini Spin Kit code 28-9042-64 (GE Healthcare, UK).

\section{Genotyping}

The PER3 length polymorphism was genotyped using the following primers flanking the VNTR: forward (5'CAAAATTTTATCACACTACCAGAATGCCTGAC-3') and reverse (5'-AACCTTGTACTTCCACATCAGTGCCTGG-3') as described elsewhere (14). For CLOCK genotyping, we used the Taqman Real Time SNP Genotyping Assay from Applied Biosystems, assay number C_8746719_20.

\section{Statistical analysis}

The allelic frequencies in the groups were compared using the chi-square test with Yates correction for comparison between two ethnic groups. The genotypic frequencies were checked for Hardy-Weinberg equilibrium. The level of significance was set at $\mathrm{P}<0.05$.

\section{Results}

The genotype distributions and allele frequency for both genes differed between the two ethnic groups (Tables 1 and 2). The frequency of the $4 / 4$ genotype in the PER3 gene was higher in the Asian than in the Caucasian group. A similar result was observed for the CLOCK gene, with the TT genotype being higher among Asians than Caucasians.

\section{Discussion}

Our results show that there are significant differences in the genetic frequencies of the VNTR polymorphism of PER3 and of T3111C of CLOCK between Asian and Caucasian populations living in Brazil. The allele frequency of 4 repeats in the PER3 gene was much higher in Asians than Caucasians and likewise the CLOCK gene T3111 allele frequency was higher in Asians than Caucasians. 
Table 1. Genotype distribution of the PER3 and CLOCK gene polymorphisms in Asian and Caucasian volunteers.

\begin{tabular}{lcccccccc}
\hline Group & \multicolumn{4}{c}{ PER3 } & & \multicolumn{3}{c}{ CLOCK } \\
\cline { 2 - 3 } \cline { 8 - 9 } & $4 / 4$ & $4 / 5$ & $5 / 5$ & & TT & TC & CC \\
\hline Asian* $(\mathrm{N}=109)$ & $81(0.74)$ & $25(0.23)$ & $3(0.03)$ & & $78(0.71)$ & $27(0.25)$ & $4(0.04)$ \\
Caucasian $(\mathrm{N}=135)$ & $63(0.47)$ & $59(0.43)$ & $13(0.10)$ & & $68(0.50)$ & $55(0.41)$ & $12(0.09)$ \\
\hline
\end{tabular}

Data are reported as number and frequency of the genotype within parentheses. *Significant difference of genotype distributions from the Caucasian group for both genes. For PER3 gene VNTR polymorphism, chi-square $=19.7$ and $P<0.0001 .4 / 4$ is the genotype homozygous for the 4 repeats, $4 / 5$ is the heterozygous genotype, and $5 / 5$ is the homozygous genotype for 5 repeats. For the CLOCK gene polymorphism $(\mathrm{T} 3111 \mathrm{C})$, chi-square $=11.6$ and $\mathrm{P}=0.003$. TT is the genotype homozygous for the $\mathrm{T}$ allele, TC is the heterozygous genotype, and CC is the homozygous genotype for the $\mathrm{C}$ allele.

In recent years, an increasing number of studies have shown association between clock gene polymorphisms and circadian phenotypes in a variety of population samples $(4,5,14)$. These remarkable results indicate a great potential for preventive medicine in terms of predicting circadian phase adaptation to temporal social demands. However, some associations described in Asian populations cannot be confirmed in Caucasians, because these polymorphisms are infrequent or missing in Caucasians $(5,7)$. Our results directly confirm the different distributions between Asian and Caucasian ethnic groups of the two polymorphisms of clock genes most frequently studied thus far.

The function of the biological clock is to supply temporal information to the organism so that physiological and/or behavioral responses can be coordinated during the daily cycle to maximize adaptability (15). However, daily fluctuations in light intensity and temperature and seasonal changes in day length vary considerably in different latitudes and locales $(16,17)$. Such changes may have contributed to the diverse patterns of polymorphisms in circadian clock genes.

Differences in allele frequencies and genotype distributions between Asians and Caucasians have been often observed in other genes (18), but, in addition to purely ethnic differences, our data are clearly linked to a strong environmental factor that drives circadian rhythmicity and therefore could act as a selection force on these polymorphisms, i.e., the daily fluctuation in light intensity along the year due to the latitudinal cline.

Based on previous studies and on the present findings $(16,17,19)$ and considering the history of human migrations around the planet over time, as well as the special characteristics of sunshine and light inherent to each place on earth, it is worth questioning which factors contributed to genetic variations that are specific to each ethnic group, especially considering the clock genes. Since the circadian phenotype is a result of strong interaction between the environment and the organism and the most important Zeitgeber for humans is the light/dark cycle given by the sun (15), it would be logical to think that differences in latitude could
Table 2. Allele frequencies of the two polymorphisms in Asian and Caucasian volunteers.

\begin{tabular}{lccccc}
\hline & \multicolumn{2}{c}{ PER3 } & & \multicolumn{2}{c}{ CLOCK } \\
\cline { 2 - 3 } \cline { 5 - 6 } & 4 repeats & 5 repeats & & T & C \\
\hline Asian* & 0.86 & 0.14 & & 0.84 & 0.16 \\
Caucasian & 0.69 & 0.31 & & 0.71 & 0.29 \\
\hline
\end{tabular}

*Significant difference of allele frequencies from the Caucasian group for both genes. For the PER3 alleles, chi-square $=18.8$ and $\mathrm{P}<0.0001$. For the Clock alleles, chi-square $=11.0$ and $\mathrm{P}$ $=0.0006$.

contribute in an important manner to the selection of these polymorphisms in different geographical zones. Studies with Drosophila have reported association of polymorphisms in clock genes with latitudinal clines (20).

It has been debated whether there is selective pressure for clock gene polymorphisms in humans. Nadkarni et al. (16) could not provide evidence that the day length or the mean annual isolation contributed as selective pressures to the VNTR polymorphism of the PER3 gene in humans. Using a similar strategy but investigating several clock and clock-related genes in five ethnic groups, Ciarleglio et al. (17) have reported that is unlikely that a pattern of natural selection has shaped the evolutionary process of these genes, suggesting that a random process is involved. On the other hand, Cruciani et al. (19) have suggested that polymorphisms in the PER2 gene might have been influenced by natural selection.

Our data suggest that, especially regarding the research field of clock genes and circadian phenotypes, ethnic variations in polymorphisms should be analyzed carefully considering the sunlight regimen of the different places on the earth where the different ethnic groups have evolved. Given that these clock genes are involved in the molecular mechanism of circadian rhythm regulation, such 
as the regulation of the endogenous period (Tau) $(1,3)$, the present finding indicates that the different profiles of the polymorphism combination may result in a different output of the molecular circadian clock among different ethnic groups. On this basis, we could ask: do Caucasian and Asian clocks tick differently?

Studies on the levels and patterns of variation in human clock genes will be very relevant for a better understand-

\section{References}

1. von Schantz M. Phenotypic effects of genetic variability in human clock genes on circadian and sleep parameters. $J$ Genet 2008; 87: 513-519.

2. Pereira DS, Tufik S, Pedrazzoli M. [Timekeeping molecules: implications for circadian phenotypes]. Rev Bras Psiquiatr 2009; 31: 63-71.

3. Konopka RJ, Benzer S. Clock mutants of Drosophila melanogaster. Proc Natl Acad Sci U S A 1971; 68: 2112-2116.

4. Katzenberg D, Young T, Finn L, Lin L, King DP, Takahashi JS, et al. A CLOCK polymorphism associated with human diurnal preference. Sleep 1998; 21: 569-576.

5. Takano A, Uchiyama M, Kajimura N, Mishima K, Inoue Y, Kamei Y, et al. A missense variation in human casein kinase I epsilon gene that induces functional alteration and shows an inverse association with circadian rhythm sleep disorders. Neuropsychopharmacology 2004; 29: 1901-1909.

6. Mishima K, Tozawa T, Satoh K, Saitoh H, Mishima Y. The $3111 \mathrm{~T} / \mathrm{C}$ polymorphism of hClock is associated with evening preference and delayed sleep timing in a Japanese population sample. Am J Med Genet B Neuropsychiatr Genet 2005; 133B: 101-104.

7. Castro RM, Barbosa AA, Pedrazzoli M, Tufik S. Casein kinase I epsilon (CKIvarepsilon) N408 allele is very rare in the Brazilian population and is not involved in susceptibility to circadian rhythm sleep disorders. Behav Brain Res 2008; 193: 156-157.

8. Pedrazzoli M, Louzada FM, Pereira DS, Benedito-Silva AA, Lopez AR, Martynhak BJ, et al. Clock polymorphisms and circadian rhythms phenotypes in a sample of the Brazilian population. Chronobiol Int 2007; 24: 1-8.

9. Takao T, Tachikawa H, Kawanishi Y, Mizukami K, Asada T. CLOCK gene T3111C polymorphism is associated with Japanese schizophrenics: a preliminary study. Eur Neuropsychopharmacol 2007; 17: 273-276.

10. Robilliard DL, Archer SN, Arendt J, Lockley SW, Hack LM, English J, et al. The 3111 Clock gene polymorphism is not associated with sleep and circadian rhythmicity in phenotypi- ing of the evolutionary mechanism that has shaped these genetic patterns in different ethnic groups.

\section{Acknowledgments}

Research supported by AFIP and FAPESP (CEPID, \#98/14303-3).

cally characterized human subjects. J Sleep Res 2002; 11 : 305-312.

11. Hohjoh H, Takasu M, Shishikura K, Takahashi Y, Honda Y, Tokunaga K. Significant association of the arylalkylamine Nacetyltransferase (AA-NAT) gene with delayed sleep phase syndrome. Neurogenetics 2003; 4: 151-153.

12. Pereira DS, Pedrazzoli M, Koike Bdel V, Louzada FM, Benedito-Silva AA, Lopez AR, et al. The G619AAa-nat gene polymorphism does not contribute to sleep time variation in the Brazilian population. Behav Genet 2007; 37: 637-638.

13. Ribeiro D. The Brazilian people: The formation and the meaning of Brazil. Florida: University Press of Florida; 2000.

14. Viola AU, Archer SN, James LM, Groeger JA, Lo JC, Skene DJ, et al. PER3 polymorphism predicts sleep structure and waking performance. Curr Biol 2007; 17: 613-618.

15. Roenneberg T, Kumar CJ, Merrow M. The human circadian clock entrains to sun time. Curr Biol 2007; 17: R44-R45.

16. Nadkarni NA, Weale ME, von Schantz M, Thomas MG. Evolution of a length polymorphism in the human PER3 gene, a component of the circadian system. J Biol Rhythms 2005; 20: 490-499.

17. Ciarleglio CM, Ryckman KK, Servick SV, Hida A, Robbins S, Wells N, et al. Genetic differences in human circadian clock genes among worldwide populations. J Biol Rhythms 2008; 23: $330-340$.

18. Allocco DJ, Song Q, Gibbons GH, Ramoni MF, Kohane IS. Geography and genography: prediction of continental origin using randomly selected single nucleotide polymorphisms. BMC Genomics 2007; 8: 68.

19. Cruciani F, Trombetta B, Labuda D, Modiano D, Torroni A, Costa R, et al. Genetic diversity patterns at the human clock gene period 2 are suggestive of population-specific positive selection. Eur J Hum Genet 2008; 16: 1526-1534.

20. Costa R, Peixoto AA, Barbujani G, Kyriacou CP. A latitudinal cline in a Drosophila clock gene. Proc Biol Sci 1992; 250: 43-44. 